\title{
Long noncoding RNA GAS5 promotes apoptosis in primary nucleus pulposus cells derived from the human intervertebral disc via Bcl-2 downregulation and caspase-3 upregulation
}

\author{
YIFENG WANG ${ }^{1}$, QINGXIN SONG ${ }^{2}$, XUAN HUANG $^{3}$, ZHI CHEN $^{2}$, FAN ZHANG $^{4}$, \\ KUN WANG $^{2}$, GUOFENG HUANG ${ }^{5}$ and HONGXING SHEN ${ }^{2}$ \\ ${ }^{1}$ Department of Orthopaedic Surgery, The First Affiliated Hospital of Xiamen University, Xiamen, Fujian 361003; \\ ${ }^{2}$ Department of Orthopaedic Surgery, Renji Hospital, Shanghai Jiao Tong University School of Medicine, \\ Shanghai 200127; ${ }^{3}$ Department of Joint Surgery, Changhai Hospital, The Second Military Medical University, \\ Shanghai 200433; ${ }^{4}$ Department of Orthopaedic Surgery, The First Affiliated Hospital of Kunming Medical University, \\ Kunming, Yunnan 650032; ${ }^{5}$ Department of Orthopaedic Surgery, No. 175 Hospital of PLA, \\ The Affiliated Southeast Hospital of Xiamen University, Zhangzhou, Fujian 363000, P.R. China
}

Received November 2, 2017; Accepted October 9, 2018

DOI: $10.3892 / \mathrm{mmr} .2019 .9883$

\begin{abstract}
Nucleus pulposus cell (NPC) apoptosis serves an important role in intervertebral disc degeneration (IDD); however, the roles of long noncoding RNAs (lncRNAs) in this process remain unknown. The present study aimed to determine the effects of the lncRNA growth arrest-specific transcript 5 (GAS5) on the apoptosis of primary human NPCs derived from the intervertebral disc, and to investigate the underlying mechanisms. TargetScan was used to predict the lncRNAs targeted by microRNA-155 (miR-155). Then, NPCs were subjected to lentivirus-mediated transduction of miR-155 or GAS5. A human lncRNA and mRNA array was used to screen differentially expressed lncRNAs following miR-155 overexpression. GAS5 and miR-155 expression levels were determined by reverse transcription-quantitative polymerase chain reaction. After GAS5 overexpression, apoptosis was assessed by flow cytometry via Annexin V/propidium iodide staining. Western blotting was employed to determine the expression of apoptosis-associated proteins, including caspase-3 and B cell lymphoma 2 (Bcl-2). TargetScan indicated GAS5 had one binding site for miR-155. Following exogenous transfection of miR-155 mimics, GAS5 expression levels in NPCs were significantly decreased $(\mathrm{P}<0.05)$. Interestingly, miR-155 overexpression in NPCs resulted in 721 differentially expressed lncRNAs compared with the negative control group $(\mathrm{P}<0.05)$,
\end{abstract}

Correspondence to: Professor Hongxing Shen, Department of Orthopaedic Surgery, Renji Hospital, Shanghai Jiao Tong University School of Medicine, 180 Pujian Road, Shanghai 200127, P.R. China E-mail: shenhxgk@126.com

Key words: long non-coding RNA growth arrest-specific transcript 5, intervertebral disc degeneration, primary nucleus pulposus cells, apoptosis, microRNA-155 including 492 and 229 upregulated and downregulated lncRNAs respectively. In addition, 18 transcripts of GAS5 exhibited a downregulated expression profile. GAS5 overexpression in NPCs resulted in enhanced caspase-3 decreased Bcl-2 expression levels; the apoptosis of NPCs was significantly increased $(\mathrm{P}<0.05)$. The results of the present study revealed that overexpression of IncRNA GAS5 may promotes NPC apoptosis via $\mathrm{Bcl}-2$ downregulation and caspase-3 upregulation, which may be associated with miR-155. The results of the present study suggest that lncRNA GAS5-silenced NPCs, or lentivirus-mediated lncRNA GAS5 knockdown may be precise and effective therapeutic strategies in the treatment of IDD.

\section{Introduction}

Intervertebral disc degeneration (IDD) is one of the major causes of lower back pain and spinal degenerative diseases. Globally, the point prevalence of lower back pain was reported to be $9.4 \%$, which ranked the highest regarding the number of years living with the condition (1). Recently, the morbidity from spinal degenerative diseases has rapidly increased with the aging of the population; at present, therapies mainly focus on alleviating clinical symptoms rather than restoring the underlying pathophysiological processes. Therefore, further investigation into IDD is urgently required (2).

Previous studies demonstrated that nucleus pulposus cell (NPC) apoptosis serves a significant role in the occurrence and development of IDD (3-6). The apoptosis of NPCs initiates degenerative cascades in the aging nucleus pulposus (NP), and leads to structural and mechanical instability of the intervertebral disc $(7,8)$. Therefore, the anti-apoptotic targeting of NPCs by molecular or cellular therapies in the intervertebral disc via percutaneous puncture may delay or reverse the process of IDD.

Noncoding RNAs, including microRNAs (miRNAs) and long noncoding RNAs (lncRNAs), regulate gene expression in numerous cells, and have been demonstrated to be highly involved in IDD (9). In NPCs, lower levels of miR-155 
expression promoted Fas-mediated apoptosis by targeting Fas-associated protein with death domain and caspase-3 (10), and decreased the amount of aggrecan and collagen type II via matrix metalloproteinase (MMP)-16 upregulation (11). Additionally, increasing evidence has suggested the important roles of lncRNAs in IDD, which may serve as novel therapeutic targets for degenerative spinal diseases $(12,13)$. In addition, the sophisticated crosstalk between miRNAs and lncRNAs also suggests their notable functions of coordinating gene expression in a multitude of processes (14); however, investigations into the interactions between miRNAs and lncRNAs in IDD are scarce.

The growth arrest-specific transcript 5 (GAS5) gene was firstly isolated by Schneider et al (15) in growth-inhibited cells in 1988 and was classified as a non-protein-coding multiple small nucleolar RNA that serves a major growth-inhibiting role (16). Numerous studies reported that GAS5 was pivotal in promoting apoptosis and suppressing cell proliferation in mammals (17-19); however, the function of GAS5 in IDD remains unknown. In the present study, IncRNAs targeted by miR-155 were screened and the lncRNA GAS5 was predicted to be a target of miR-155 in NPCs. Therefore, the functional mechanism of GAS5 was investigated to reveal the effects of GAS5 on primary human NPC apoptosis. Interestingly, GAS5 overexpression promoted NPC apoptosis via B cell lymphoma 2 (Bcl-2) downregulation and caspase-3 upregulation. The findings of the present study indicated that GAS5 may be a novel therapeutic target for IDD due to its pro-apoptotic effects.

\section{Materials and methods}

Cell culture. Intervertebral disc-derived primary human NPCs were obtained from ScienCell Research Laboratories, Inc. (cat. no. 4800) $(20,21)$, and cultured in NPC medium (cat. no. 4801; both ScienCell Research Laboratories, Inc., San Diego, CA, USA) at $37^{\circ} \mathrm{C}$ in a humidified atmosphere containing $5 \% \mathrm{CO}_{2}$. At $\sim 80-90 \%$ confluence, cells were passaged at a ratio of $\sim 1: 2-3$.

Prediction of miR-155 targeting lncRNAs by TargetScan. A total of three miRNA target gene prediction software programs were compared, including TargetScan version 7.0 (http://www.targetscan.org/), PITA version 6 (https://omictools.com/pita-tool) and PicTar (https://pictar.mdc-berlin.de/); analysis with TargetScan was selected for its high sensitivity and accuracy in analyzing non-conserved domains $(22,23)$. The miRNAs targeting lncRNAs were screened based on the method of conserved seed pairing via TargetScan analysis; however, during the prediction of miRNA-targeted lncRNAs, the TargetScan Perl script was run by replacing the mRNA sequences with lncRNA sequences. In the present study, NPC apoptosis-associated lncRNAs were screened by searching the IncRNAs targeted by miR-155. miR-155 expression is decreased in the degenerative NP $(10,11)$; 866 upregulated lncRNAs were confirmed as subjects for analysis in the present study. Additionally, retrieval with TargetScan version 7.0, Ensembl Genome Browser (http://asia.ensembl. org/index.html), UCSC Genome Browser (http://genome. ucsc.edu/), and miRBase release 21 (http://www.mirbase. org/) further confirmed the sequence of 631 lncRNAs used for bioinformatics analysis.
Transfection of miR-155 mimics. The sequence of mature hsa-miR-155-5p (MIMAT0000646) was retrieved from the miRBase database (http://www.mirbase.org/). Double-chain hsa-miR-155-5p and double-chain negative control (NC) RNA were designed and synthesized by Shanghai GenePharma Co., Ltd.(Shanghai,China). The synthesized hsa-miR-155-5p mimics were: Sense, 5'-UUAAUGCUAAUCGUGAUAGGGGU-3' and antisense, 5'-CCCUAUCACGAUUAGCAUUAAUU-3'. The double-chain NC RNA sequences were: Sense, 5'-UUCUCC GAACGUGUCACGUTT-3' and antisense, 5'-ACGUGACAC GUUCGGAGAATT-3'. The day prior to transfection, NPCs were seeded into a 24 -well plate at a density of $0.5 \times 10^{5} /$ well in $500 \mu \mathrm{l}$ NPC medium. At $\sim 70 \%$ confluence, NPCs were transfected with $1 \mu \mathrm{l}$ double-chain hsa-miR-155-5p $(20 \mu \mathrm{M})$ and double-chain NC RNA $(20 \mu \mathrm{M})$, respectively, using Lipofectamine ${ }^{\circledR} 2000$ (Invitrogen; Thermo Fisher Scientific, Inc., Waltham, MA, USA). After $72 \mathrm{~h}$ of incubation at $37^{\circ} \mathrm{C}$, the cells were assessed by reverse transcription-quantitative polymerase chain reaction (RT-qPCR) as described below.

Lentivirus-mediated hsa-miR-155 overexpression. Lentivirus-mediated miR-155 overexpression reagents were ordered from Shanghai (Lingke) Biotechnology Co., Ltd. (Shanghai, China), Packaging 293T cells were transfected with plasmids comprised of pRsv-REV, pMDlg-pRRE, pMD2G and transfer vector: pLenO-GTP. The NPCs transfected miR-155 overexpression exhibited green fluorescence under fluorescence microscopy due to the element of pLenO-GTP expressing green fluorescence protein. Using fluorescence microscopy (magnification, x40; Olympus IX73-DP80; Olympus Corporation, Tokyo, Japan), the efficiency of transduction was evaluated by calculating the percentage of green fluorescent protein-positive NPCs in 10 fields of view near the center of the culture vessel. The day prior to transduction, NPCs were seeded into a 6-well plate at a density of $5.0 \times 10^{5} /$ well in $2.5 \mathrm{ml} \mathrm{NPC} \mathrm{medium} \mathrm{and}$ incubated under a humidified atmosphere with $5 \% \mathrm{CO}_{2}$ at $37^{\circ} \mathrm{C}$. At $\sim 60 \%$ confluence, NPCs were transduced with miR-155 or a NC at a multiplicity of infection (MOI) of 80. After $96 \mathrm{~h}$ post-incubation, total RNA was extracted and assessed via an lncRNA array as described below. The efficiency of transduction was determined successful when $>95 \%$ at $96 \mathrm{~h}$ after transduction.

Human lncRNA plus mRNA array. Total RNA was extracted using TRIzol ${ }^{\circledR}$ reagent (Invitrogen; Thermo Fisher Scientific, Inc.) from lentivirus-mediated miR-155 overexpression NPCs and NC groups. The purity and concentration, as determined by the optical density (OD) $260 / \mathrm{OD} 280$ ratio, was $\geq 1.90$ with total RNA $\geq 1 \mu \mathrm{g}$ per sample, which was assessed with a NanoDrop 1000 device (NanoDrop Technologies; Thermo Fisher Scientific, Pittsburgh, PA, USA). Denaturing formaldehyde agarose gel electrophoresis (percentage of 1.2\%) demonstrated clear RNA bands, with $28 \mathrm{~S}$ to $18 \mathrm{~S}$ exceeding 2 bands and good RNA integrity (data not shown). Then, lncRNA array detection was performed by Beijing Capitalbio Technology Co., Ltd. (Beijing, China).

The Agilent human lncRNA + mRNA Array v4.0 (Agilent Technologies, Inc., Santa Clara, CA, USA) was used to detect lncRNAs in the present model system. Cy5 and Cy3 were used to label the molecule types on the same array at $37^{\circ} \mathrm{C}$ for $1.5 \mathrm{~h}$ 
and $70^{\circ} \mathrm{C}$ for $5 \mathrm{~min}$, respectively. The Agilent array contained probes capable of detecting 41,000 human lncRNAs and 34,000 human mRNAs, in a $4 \times 180 \mathrm{~K}$ format. The array also contained 4,974 internal Agilent control probes. The experiments were performed according to the manufacturer's protocols and conducted twice. The fluorescent signals of $\mathrm{Cy} 3$ and $\mathrm{Cy} 5$ were obtained, and differentially expressed mRNAs and lncRNAs were screened, at a fold change cutoff of 2 and $\mathrm{P}<0.05$.

Lentivirus-mediated GAS5 overexpression. Lentivirus-mediated GAS5 overexpression reagents were ordered from Lingke Biotechnology Co., Ltd., by using packaging 293T cells transfected with plasmids comprised of pRsv-REV, pMDlg-pRRE, pMD2G and transfer vector: pLenO-GTP. The NPCs with GAS5 overexpression exhibited green fluorescence for the element of pLenO-GTP expressing green fluorescence protein. NPCs were seeded into a 6-well plate at a density of $5.0 \times 10^{5} /$ well in $2.5 \mathrm{ml} \mathrm{NPC}$ medium and incubated for $24 \mathrm{~h}$ under a humidified atmosphere with $5 \% \mathrm{CO}_{2}$ at $37^{\circ} \mathrm{C}$. At $\sim 60 \%$ confluence, NPCs were transduced with GAS5 (NR_002578.2; https://www.ncbi .nlm.nih.gov/nuccore/NR_002578.2) or NC (empty vector) at an MOI of 80. After $96 \mathrm{~h}$ post-incubation, GAS5 expression was assessed by RT-qPCR.

$R T-q P C R$. Total RNA was extracted from NPCs using TRIzol reagent; the purity and quantity of RNA were assessed using a NanoDrop 1000 system. RT was performed on an ABI Veriti gradient PCR system (Applied Biosystems; Thermo Fisher Scientific, Inc.) using a PrimeScript ${ }^{\mathrm{TM}}$ RT-PCR kit (Takara Bio, Inc., Otsu, Japan), the reaction conditions were: $30^{\circ} \mathrm{C}$ for $10 \mathrm{~min}, 42^{\circ} \mathrm{C}$ for $30 \mathrm{~min}$ and $72^{\circ} \mathrm{C}$ for $15 \mathrm{~min}$. The following primers were used: Hsa-miR-155-5p forward, 5'-GGGGGT AATGCTAATCGTGAT-3' and reverse, 5'-GTGCGTGTC GTGGAGTCG-3'; U6 forward, 5'-GCTTCGGCAGCACAT ATACTAAAAT-3' and reverse, 5'-CGCTTCACGAATTTG CGTGTCAT-3'; 1ncRNA GAS5 sense, 5'-GCTTACTGC TTGAAAGGGTCT-3' and antisense, 5'-CACTGGGAGGCT GAGGAT-3'; $\beta$-actin sense, 5'-GCACCACACCTTCTACAA TGAG-3' and antisense, 5'-ACAGCCTGGATAGCAACG T-3'. qPCR was performed on a Light Cycler 480 II RT-PCR system (Roche Diagnostics, Indianapolis, IN, USA) with SYBR-Green I (Takara Bio, Inc.). The reaction conditions were: $95^{\circ} \mathrm{C}$ for $30 \mathrm{sec}, 95^{\circ} \mathrm{C}$ for $5 \mathrm{sec}$ and $60^{\circ} \mathrm{C}$ for $30 \mathrm{sec}$ (40 cycles). Each experiment was repeated three times, the relative mRNA expression levels were evaluated by the $2^{-\Delta \Delta C q}$ method (24).

Cell apoptosis detection by flow cytometry. NPCs exhibited green fluorescence following lentiviral transduction, Annexin V-allophycocyanin (eBioscience; Thermo Fisher Scientific, Inc.) was used for cytomembrane staining (NPCs were stained for $25 \mathrm{~min}$ at room temperature without light), and propidium iodide (eBioscience; Thermo Fisher Scientific, Inc.) was used for nuclear staining (NPCs were stained for $5 \mathrm{~min}$ at room temperature). Subsequently, the transfection efficiency and apoptosis of NPCs were assessed using a CytoFLEX flow cytometer (Beckman Coulter, Inc., Brea, Ca, USA). Data were analyzed via the FlowJo 7.6.1 software (FlowJo LLC, Ashland, OR, USA). NPCs stained with Annexin V-allophycocyanin without propidium iodide were considered as early apoptotic NPCs, and the early apoptosis rate was determined by calculating the percentage of NPCs in the lower right quadrant of the scatter diagram.

Western blotting. Total protein from NPCs was extracted following lysis with radioimmunoprecipitation assay buffer (Thermo Fisher Scientific, Inc.), and quantified with a Bicinchoninic Acid protein assay. Then, $40 \mu \mathrm{g}$ total protein from NPCs were loaded and separated by $15 \%$ SDS-PAGE and transferred onto polyvinylidene difluoride membranes (Merck KGaA, Darmstadt, Germany). Following blocking with $5 \%$ bovine serum albumin for $2 \mathrm{~h}$ at room temperature, rabbit anti-human caspase-3, rabbit anti-human Bcl-2 and mouse anti-human $\beta$-actin polyclonal antibodies (cat. nos. 9665, 2870 and 3700; 1:1,000; Cell Signaling Technology, Inc., Danvers, MA, USA) were added respectively overnight at $4^{\circ} \mathrm{C}$. Then, membranes were washed with tris-buffered saline with $0.1 \%$ Tween-20 and incubated with anti-rabbit secondary antibodies conjugated to horseradish peroxidase (cat. no. 7074; 1:1,000) for rabbit anti-human caspase-3 and rabbit anti-human Bcl-2 antibodies and anti-mouse secondary antibodies conjugated to horseradish peroxidase for mouse anti-human $\beta$-actin antibody (cat. no. 7076; 1:1,000; both Cell Signaling Technology, Inc.). Finally, the membranes were developed with an enhanced chemiluminescence reagent (Pierce ${ }^{\mathrm{TM}}$ ECL Western Blotting Substrate, Pierce; Thermo Fisher Scientific, Inc.); protein bands were imaged under a FluorChem FC2 visualizer (Alpha Innotech, San Leandro, CA, USA).

Statistical analysis. SSPSS 19.0 (IBM Corp., Armonk, NY, USA) was used for data analysis. Data were presented as the mean \pm standard deviation from three independent experiments performed in triplicate. Comparisons between groups were conducted using an Independent Samples t-test. $\mathrm{P}<0.05$ was considered to indicate a statistically significant difference.

\section{Results}

Overexpression of miR-155 in NPCs. NPCs were transduced with lentiviral vectors (Fig. 1). Under fluorescence microscopy, the transduction efficiencies were determined to be $>95 \%$ (Fig. 1A). In addition, miR-155 expression levels in lentiviral-transduced NPCs were assessed by RT-qPCR; significantly higher miR-155 expression levels were detected in NPCs transduced with miR-155 compared with the control group (Fig. 1B). These findings indicated a highly efficient and successful overexpression of miR-155 in transduced NPCs.

Prediction of miR-155 targeted lncRNAs. TargetScan data analysis revealed that among the 631 upregulated lncRNAs with defined sequences, there were 148 differentially expressed IncRNAs with 178 hsa-miR-155-5p binding sites in total (data not shown). Among these, NR_002819 with 8,707 nucleotides, which included 6 hsa-miR-155-5p binding sites; ENST00000511037 and NR_027451 contained 1801 and 5343 nucleotides, respectively, which included 5 hsa-miR-155-5p binding sites. In addition, there were 19 lncRNAs with 2 hsa-miR-155-5p binding sites and 126 IncRNAs containing 

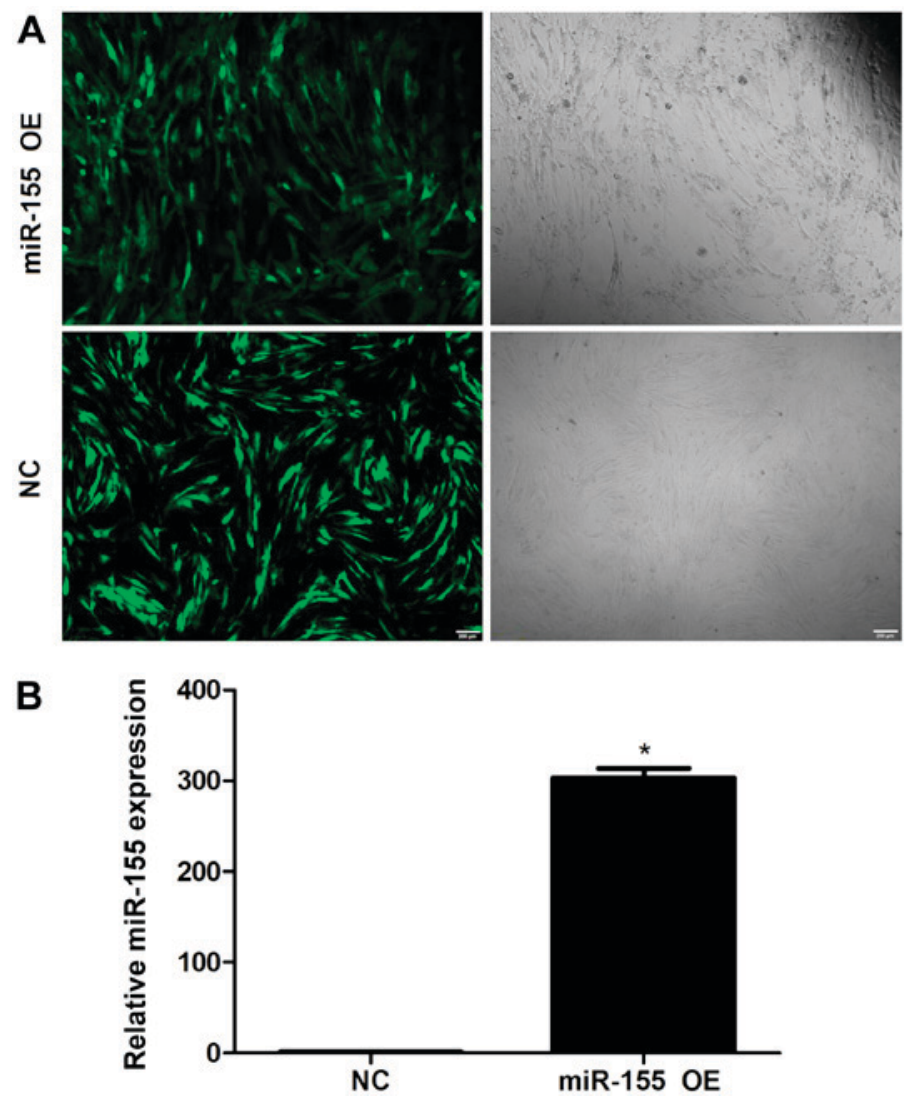

Figure 1. Expression of miR-155 in nucleus pulposus cells following transduction with miR-155 OE lentiviruses. (A) Transduction efficiencies of green fluorescent protein-positive cells was determined successful when $>95 \%$ at $96 \mathrm{~h}$ after transduction. Magnification, $\mathrm{x} 40$. (B) miR-155 expression levels determined by reverse transcription-quantitative polymerase chain reaction at $96 \mathrm{~h}$ following transduction. Data are presented as the mean \pm standard error of the mean from three independent experiments performed in triplicate. " $\mathrm{P}<0.05$ vs. NC group. miR, microRNA; NC, negative control; OE, overexpression.
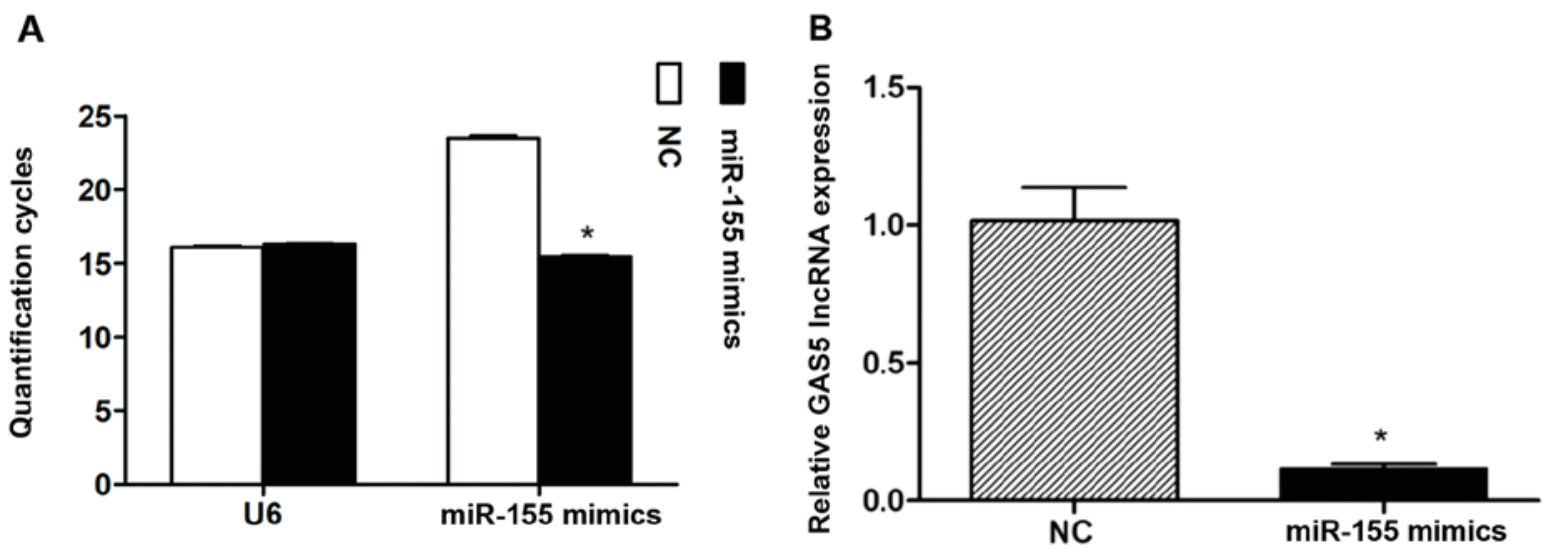

Figure 2. GAS5 expression levels after $72 \mathrm{~h}$ following nucleus pulposus cell transfection with miR-155 mimics. (A) miR-155 transfection was confirmed via reverse transcription-quantitative polymerase chain reaction. Data are presented as the mean \pm standard error of the mean from three independent experiments performed in triplicate. ${ }^{*} \mathrm{P}<0.05$ vs. NC group. (B) GAS5 expression levels were determined following transfection with miR-155 mimics. Data are presented as the mean \pm standard deviation from three independent experiments performed in triplicate. " $\mathrm{P}<0.05$ vs. NC group. GAS5, growth arrest-specific transcript 5 ; miR, microRNA; NC, negative control.

1 hsa-miR-155-5p binding site. Furthermore, NR_002578 was observed to contain a hsa-miR-155-5p binding site and was also predicted to be a target of miR-155. Additionally, a literature review indicated that NR_002578 is an lncRNA transcribed from GAS5 and is highly expressed in NPCs of the degenerative intervertebral disc (12), with a tumor suppressor role in a variety of tumors, promoting tumor cell apoptosis (19).
miR-155 overexpression inhibits GAS5 expression in NPCs. After $\sim 72 \mathrm{~h}$ following miR-155 mimics transfection into NPCs (Fig. 2A), the overall GAS5 expression levels were determined. The results revealed significantly lower expression levels of GAS5 compared with the NC group (Fig. 2B).

LncRNA array analysis in NPCs overexpressing miR-155. LncRNA array analysis revealed 721 differentially expressed 
lncRNAs following transduction with miR-155 lentiviruses, 492 upregulated and 229 downregulated lncRNAs, respectively (Fig. 3). A total of 18 GAS5 transcripts were detected, and each individual transcript showed downregulated expression (Table I); however, the fold changes of each individual transcript did not exhibit significance. The results suggested that different overexpression mehtods using mimics or lentiviruses may affect GAS5 expression.

Effects of GAS5 overexpression on cell apoptosis and apoptosis-associated proteins in NPCs. The transduction efficiency was determined to be $95 \%$ at $96 \mathrm{~h}$ post-transduction (Fig. 4A), and classic bleb-like protuberances were observed during cell growth (Fig. 4B). At $96 \mathrm{~h}$ following lentiviral transduction, GAS5 expression levels were significantly increased compared with the NC group ( $\mathrm{P}<0.05$; Fig. 4C).

As detected by flow cytometry, the early apoptosis rates were $35.4 \pm 1.86$ and $42.6 \pm 2.23 \%$ in the NC and GAS5 overexpression groups, respectively, which indicated that GAS5 overexpression induced early apoptosis of NPCs $(\mathrm{P}<0.05$; Fig. 5A). The expression levels of the two apoptosis-associated proteins, caspase- 3 and $\mathrm{Bcl}-2$, which were determined by western blotting, were notably upregulated and downregulated, respectively, when GAS5 was overexpressed in NPCs (Fig. 5B).

\section{Discussion}

Numerous lncRNAs are differentially expressed in NP tissues, which suggests the important roles of lncRNAs in the development of IDD $(12,13)$. Previous studies have preliminarily investigated several functional mechanisms of IncRNA GAS5, mostly in neoplastic and osteoarthritis degenerative diseases. Song et al (25) reported that GAS5 overexpression increased the expression levels of MMP-2, MMP-3, MMP-9, MMP-13 and A disintegrin and metalloproteinase with thrombospondin motifs- 4 in cartilage cells, further inducing apoptosis and inhibiting autophagy. These effects may be inhibited by miR-21, which specifically targets GAS5. Li et al (26) demonstrated that GAS5 ameliorated LPS-induced inflammatory injury in ATDC5 chondrocytes by inhibiting the nuclear factor- $\mathrm{kB}$ and Notch signaling pathways. Additionally, the roles of GAS5 lncRNA in tumor growth inhibition were investigated in several studies. Guo et al (27) reported that GAS5 significantly increased the expression of phosphatase and tensin homolog via miR-103 inhibition, promoting the apoptosis of endometrial cancer cells. Zhao et al (28) also indicated that GAS5 inhibited glioma cell growth by directly targeting and inhibiting miR-222; however, to the best of our knowledge, no studies on cartilage tumors have been conducted.

In the present study, GAS5 was predicted as a target of miR-155 by lncRNA-miRNA regulatory network analysis and the overall expression of GAS5 was observed to be significantly reduced following the transfection of NPCs with miR-155 mimics. Furthermore, array analysis revealed that each detected GAS5 transcript exhibited a downregulated expression profile following NPC transduction with miR-155 lentiviruses. These findings indicated a negative regulatory effect of miR-155 on GAS5 expression in NPCs;
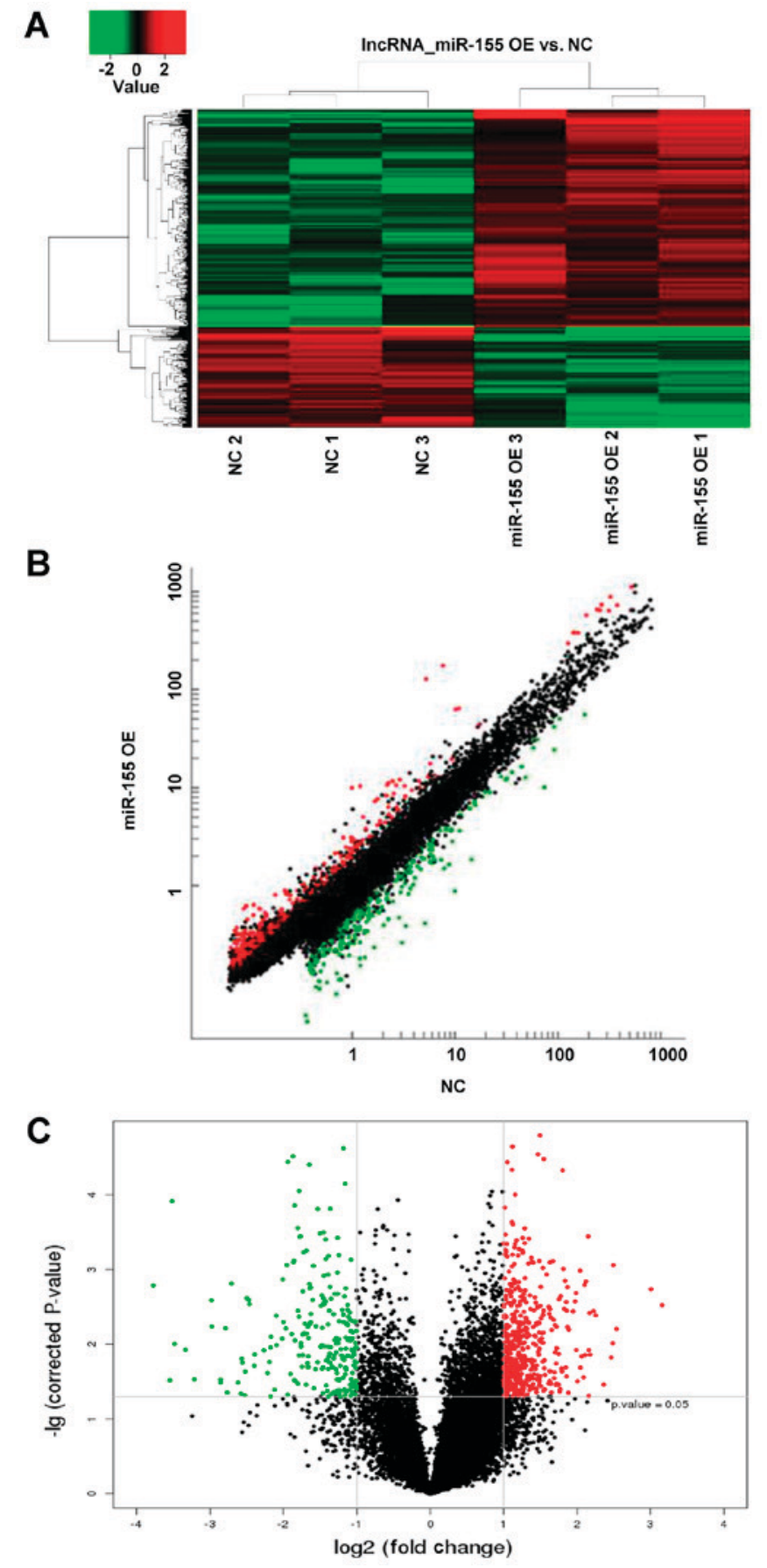

Figure 3. LncRNA array analysis. A total of 492 lncRNAs were upregulated and 229 lncRNAs were downregulated. (A) Cluster analysis of differentially expressed lncRNAs; (B) scatter plot of differentially expressed lncRNAs and (C) volcano plot of differentially expressed lncRNAs. LncRNA, long noncoding RNA; miR, microRNA; NC, negative control; OE, overexpression.

however, the underlying regulatory mechanism requires further investigation. For example, a dual-luciferase reporter gene assay may demonstrate the interaction between GAS5 with miR-155 and an RNA pull-down assay may detect the target proteins of GAS5; such investigations may clarify the functions of GAS5.

Studies on a variety of cancers demonstrated that GAS5 was a tumor suppressor, and inhibited proliferation and promoted apoptosis; GAS5 was proposed to be involved in numerous key regulatory pathways associated cell survival (29-31). Therefore, 
Table I. Expression of 29 transcripts of GAS5 lncRNA when overexpressing miR-155 in NPCs.

\begin{tabular}{|c|c|c|c|c|c|}
\hline Name & Transcript ID & Probe & Expression & $\mathrm{P}$-value & Fold change \\
\hline GAS5-001 & ENST00000450589 & $-{ }^{\mathrm{a}}$ & - & - & - \\
\hline GAS5-002 & ENST00000431268 & p28959 & Downregulated & 0.38 & 1.12 \\
\hline GAS5-003 & ENST00000448718 & - & - & - & - \\
\hline GAS5-004 & ENST00000436656 & p33995_v4 & Downregulated & 0.26 & 1.17 \\
\hline GAS5-005 & ENST00000458220 & p547 & Downregulated & - & - \\
\hline GAS5-006 & ENST00000421068 & p545 & Downregulated & 0.76 & 1.08 \\
\hline GAS5-007 & ENST00000456293 & p543 & Downregulated & 0.43 & 1.15 \\
\hline GAS5-008 & ENST00000449289 & - & - & - & - \\
\hline GAS5-009 & ENST00000455838 & p541 & Downregulated & 0.45 & 1.13 \\
\hline GAS5-010 & ENST00000449589 & - & - & - & - \\
\hline GAS5-011 & ENST00000443799 & p544 & Downregulated & 0.66 & 1.08 \\
\hline GAS5-012 & ENST00000416952 & p542 & Downregulated & 0.49 & 1.12 \\
\hline GAS5-013 & ENST00000452197 & - & - & - & - \\
\hline GAS5-014 & ENST00000454068 & p33998_v4 & Downregulated & 0.19 & 1.36 \\
\hline GAS5-015 & ENST00000451607 & p34000_v4 & Downregulated & 0.09 & 1.35 \\
\hline GAS5-016 & ENST00000432536 & p34001_v4 & Downregulated & 0.20 & 1.31 \\
\hline GAS5-017 & ENST00000436285 & - & - & - & - \\
\hline GAS5-018 & ENST00000442067 & p540 & Downregulated & 0.07 & 1.53 \\
\hline GAS5-019 & ENST00000422183 & p33997_v4 & Downregulated & 0.30 & 1.24 \\
\hline GAS5-020 & ENST00000425771 & p546 & Downregulated & 0.19 & 1.27 \\
\hline GAS5-021 & ENST00000422008 & p539 & Downregulated & 0.02 & 1.77 \\
\hline GAS5-022 & ENST00000412059 & - & - & - & - \\
\hline GAS5-023 & ENST00000422207 & - & - & - & - \\
\hline GAS5-024 & ENST00000434796 & p33999_v4 & Downregulated & 0.27 & 1.21 \\
\hline GAS5-025 & ENST00000444470 & - & - & - & - \\
\hline GAS5-026 & ENST00000454813 & p538 & Downregulated & 0.36 & 1.13 \\
\hline GAS5-027 & ENST00000430245 & p33996_v4 & Downregulated & 0.32 & 1.18 \\
\hline GAS5-028 & ENST00000414075 & - & - & - & - \\
\hline GAS5-029 & ENST00000456812 & - & - & - & - \\
\hline
\end{tabular}

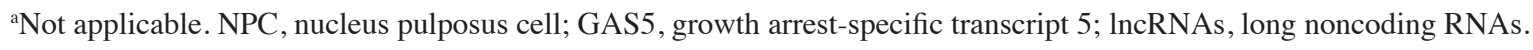

the function of GAS5 in determining cell survival or death has attracted increasing attention, particularly in cancer research (19). Consistently, a similar effect was observed in NPCs, as GAS5 overexpression promoted NPC apoptosis in vitro.

The three main apoptotic pathways (mitochondrial, death receptor and endoplasmic reticulum stress pathways) are involved in different stages of IDD development (32). Among them, the mitochondrial pathway serves a major role in moderate to severe stages of IDD $(33,34)$. The release of apoptosis-associated molecules, including cytochrome $c$ (Cyt $c$ ), apoptosis-inducing factor, Endo G and Smac in mitochondria, is initiated by stress and apoptosis signaling; Cyt $c$ then binds apoptotic protease activating factor-1 and caspase- 9 zymogen forming the apoptosome, and activates the caspase- 3 cascade that ultimately leads to apoptosis (35). Bcl-2 and caspase proteases are two protein families with conserved evolution. Bcl-2 protein controls mitochondrial integrity and inhibits Cyt $c$ release, therefore inhibiting apoptosis (35). In addition, caspases initiate and execute the process of apoptosis; among them, caspases-3, 6, and 7 execute the process of apoptosis (35).
For instance, in vitro silencing of caspase-3 suppressed the apoptosis of NPCs induced by mechanical overload, further inhibiting IDD (36). In the present study, the effect of GAS5 on NPC apoptosis was reported, and the underlying mechanism was investigated. As aforementioned, GAS5 overexpression downregulated the expression of Bcl-2 and upregulated that of caspase-3, therefore promoting NPC apoptosis. Of note, GAS5 involvement in mitochondrial apoptosis has been previously reported; by directly targeting miR-222, GAS5 could indirectly inhibit Bcl-2 expression in glioma cells (28). In addition, GAS5 promoted the apoptosis of ovarian cancer cells via caspase- 3 and caspase-9 upregulation (37). Additionally, the oncogenesis of some cartilage tumors, including chondrosarcoma and chondroblastic osteosarcoma, has exhibited a high correlation with the mitochondrial pathway of apoptosis (38-40). Future studies on these tumors are required to further verify the function of GAS5 in mitochondrial apoptosis, providing that GAS5, as a tumor suppressor, may inhibit tumor growth and proliferation.

Biomolecular therapy, cell therapy, tissue-engineered construction and annulus fibrosus repair are novel strategies 

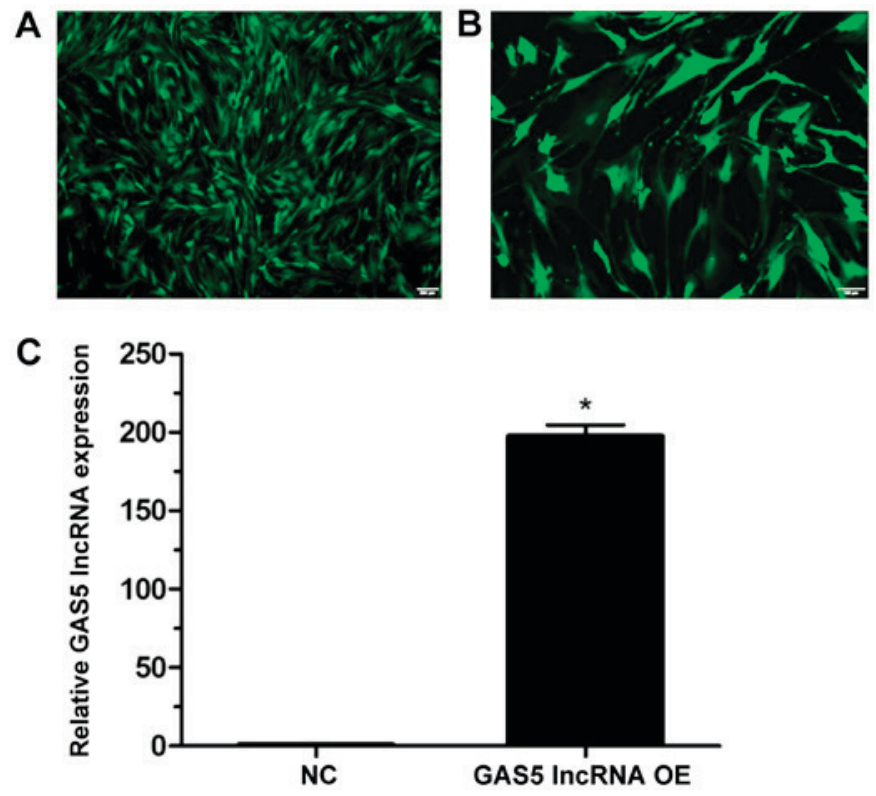

Figure 4. GAS5 expression levels following the transduction of NPCs with lentivirus containing GAS5 lncRNA. (A) Efficiency obtained $96 \mathrm{~h}$ following transduction. Magnification, x40. (B) Protuberances of NPCs observed during cell growth. Magnification, x100. (C) GAS5 expression levels assessed by reverse transcription-quantitative polymerase chain reaction at $96 \mathrm{~h}$ following transduction. Data are presented mean \pm standard deviation from three independent experiments performed in triplicate. " $\mathrm{P}<0.05$ vs. NC group. GAS5, growth arrest-specific transcript 5; lncRNA, long noncoding RNA; NC, negative control; NPCs, nucleus pulposus cells; OE, overexpression.

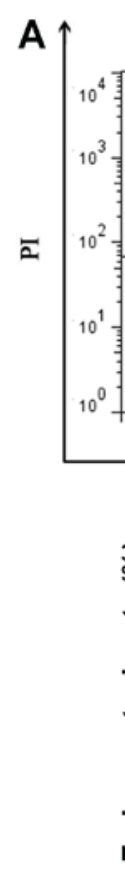

B
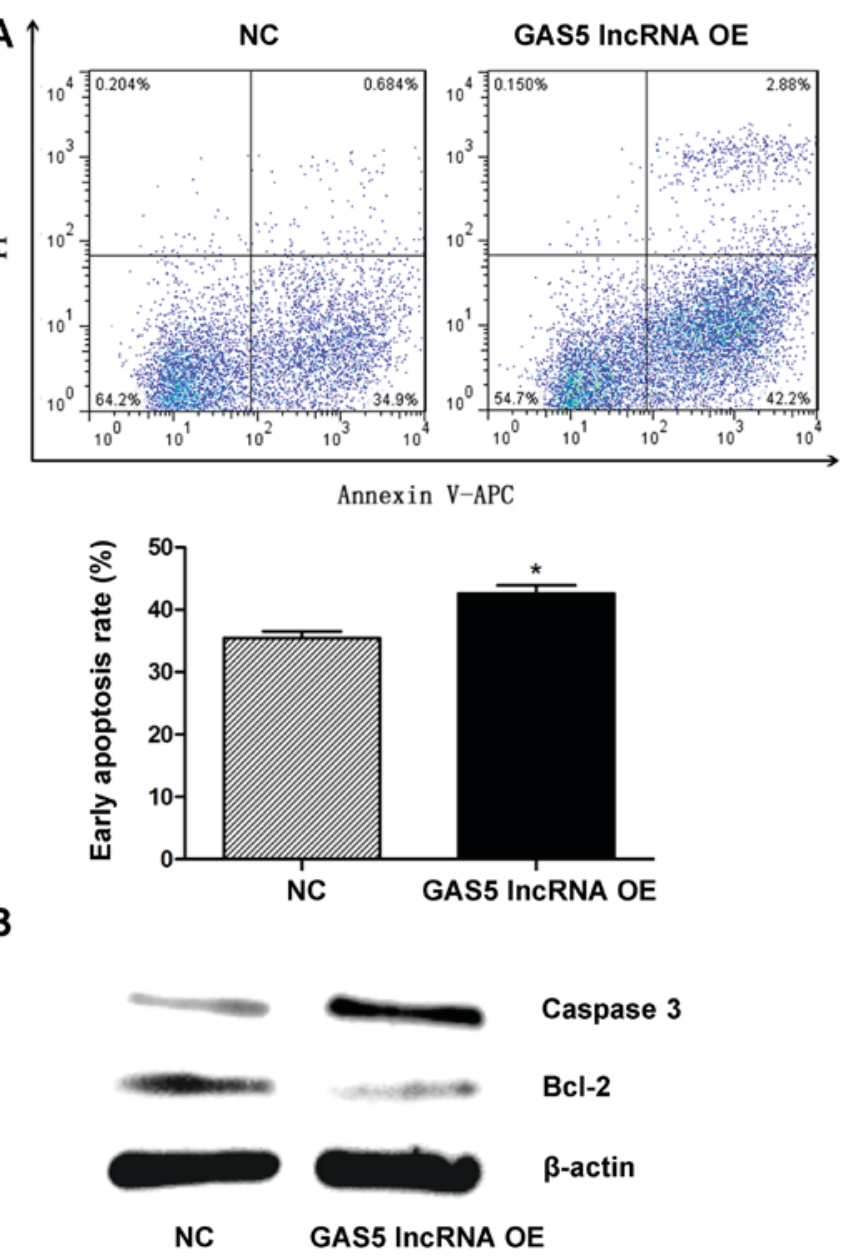

Figure 5. Effects of GAS5 OE on apoptosis and apoptosis-associated protein expression levels in nucleus pulposus cells. (A) Cell apoptosis was assessed by flow cytometry via Annexin V-APC/PI staining at $96 \mathrm{~h}$ following transduction; (B) caspase-3 and Bcl-2 protein expression levels were determined by western blotting at $96 \mathrm{~h}$ following transduction, with $\beta$-actin as a loading control. Data are presented as the mean \pm standard deviation from three independent experiments. "P<0.05 vs. NC group. APC, allophycocyanin; Bcl-2, B-cell lymphoma 2; GAS5, growth arrest-specific transcript 5; lncRNA, long noncoding RNA; NC, negative control; OE, overexpression; PI, propidium, iodide. 
for treating IDD (41). Using biomolecules, including recombinant genes, proteins or platelet-rich plasma, to adjust cellular metabolism and extracellular matrix regeneration, early degenerated disks with sufficient viable NPCs can be treated to mitigate the progression of disc degeneration (42). Midstage degeneration, characterized by fewer active cells, can be treated with cell implantations, including stem cells or allogeneic NPCs to meet the demand of the disk for active NPCs and reverse the degenerative process $(43,44)$. Accordingly, with a thorough understanding of GAS5 functions, ultrasound- or computed tomography-guided injection of GAS5-silenced NPCs, or lentivirus-mediated GAS5 knockdown may improve the precision and efficiency of IDD treatment.

There were several limitations of the present study. Firstly, GAS5 overexpression in degenerative NP, though demonstrated in a previous microarray study (12), requires further clinical investigation. In addition, RNA sample extraction in severely degenerated NP is yet to be resolved. The downregulation of GAS5 may have more clinical value in the treatment of IDD, while the present study only conducted overexpression. Additionally, the inter-regulatory dependence of the mechanism investigated in the present study requires further study.

In conclusion, GAS5 overexpression resulted in increased apoptosis in primary NPCs from the human intervertebral disc via Bcl-2 downregulation and caspase-3 upregulation. GAS5, predicted to be a target of miR-155 by TargetScan analysis, was proposed to be downregulated by miR-155 overexpression. The findings of the present study may provide novel insight into the role of GAS5 in IDD pathogenesis and contribute to developments in the treatment of IDD via biomolecular or cellular therapies.

\section{Acknowledgements}

Not applicable.

\section{Funding}

The present study was supported by the National Nature Science Foundation of China (grant no. 81672203).

\section{Availability of data and materials}

The datasets used and/or analyzed during the current study are available from the corresponding author on reasonable request.

\section{Authors' contributions}

YW, QS and HS made substantial contributions to the design of the present study. YW, QS and XH performed the majority of the experiments, data collection, statistical analysis, and wrote the manuscript. ZC, FZ, KW and GH analyzed the data and revised the manuscript. All authors reviewed the manuscript.

\section{Ethics approval and consent to participate}

Not applicable.

\section{Patient consent for publication}

Not applicable.

\section{Competing interests}

The authors declare that they have no competing interest.

\section{References}

1. Hoy D, March L, Brooks P, Blyth F, Woolf A, Bain C, Williams G, Smith E, Vos T, Barendregt J, et al: The global burden of low back pain: Estimates from the global burden of disease 2010 study. Ann Rheum Dis 73: 968-974, 2014.

2. Vo NV, Hartman RA, Patil PR, Risbud MV, Kletsas D, Iatridis JC, Hoyland JA, Le Maitre CL, Sowa GA and Kang JD: Molecular mechanisms of biological aging in intervertebral discs. J Orthop Res 34: 1289-1306, 2016.

3. Luoma K, Riihimäki H, Luukkonen R, Raininko R, Viikari-Juntura $\mathrm{E}$ and Lamminen A: Low back pain in relation to lumbar disc degeneration. Spine (Phila Pa 1976) 25: 487-492, 2000.

4. Le Maitre CL, Pockert A, Buttle DJ, Freemont AJ and Hoyland JA: Matrix synthesis and degradation in human intervertebral disc degeneration. Biochem Soc Trans 35: 652-655, 2007.

5. Liu Z, Ma C, Shen J, Wang D, Hao J and Hu Z: SDF-1/CXCR4 axis induces apoptosis of human degenerative nucleus pulposus cells via the NF-kB pathway. Mol Med Rep 14: 783-789, 2016.

6. Shen J, Fang J, Hao J, Zhong X, Wang D, Ren H and Hu Z: SIRT1 inhibits the catabolic effect of IL-1 $\beta$ through TLR2/SIRT1/NF- $\mathrm{BB}$ pathway in human degenerative nucleus pulposus cells. Pain physician 19: E215-E226, 2016.

7. Freemont AJ: The cellular pathobiology of the degenerate intervertebral disc and discogenic back pain. Rheumatology (Oxford) 48: 5-10, 2009.

8. Urban JP and Roberts S: Degeneration of the intervertebral disc. Arthritis Res Ther 5: 120-130, 2003.

9. Li Z, Yu X, Shen J, Chan MT and Wu WK: MicroRNA in intervertebral disc degeneration. Cell Prolif 48: 278-283, 2015.

10. Wang HQ, Yu XD, Liu ZH, Cheng X, Samartzis D, Jia LT, Wu SX, Huang J, Chen J and Luo ZJ: Deregulated miR-155 promotes Fas-mediated apoptosis in human intervertebral disc degeneration by targeting FADD and caspase-3. J Pathol 225: 232-242, 2011.

11. Zhang WL, Chen YF, Meng HZ, Du JJ, Luan GN, Wang HQ, Yang MW and Luo ZJ: Role of miR-155 in the regulation of MMP-16 expression in intervertebral disc degeneration. J Orthop Res 35: 1323-1334, 2017.

12. Wan ZY, Song F, Sun Z, Chen YF, Zhang WL, Samartzis D, Ma CJ, Che L, Liu X, Ali MA, et al: Aberrantly expressed long noncoding RNAs in human intervertebral disc degeneration: A microarray related study. Arthritis Res Ther 16: 465, 2014.

13. Chen Y, Ni H, Zhao Y, Chen K, Li M, Li C, Zhu X and Fu Q: Potential role of lncRNAs in contributing to pathogenesis of intervertebral disc degeneration based on microarray data. Med Sci Monit 21: 3449-3458, 2015.

14. Furió-Tarí P, Tarazona S, Gabaldón T, Enright AJ and Conesa A: SpongeScan: A web for detecting microRNA binding elements in lncRNA sequences. Nucleic Acids Res 44: W176-W180, 2016.

15. Schneider C, King RM and Philipson L: Genes specifically expressed at growth arrest of mammalian cells. Cell 54: 787-793, 1988.

16. Smith CM and Steitz JA: Classification of gas5 as a multi-small-nucleolar-RNA (snoRNA) host gene and a member of the 5'-terminal oligopyrimidine gene family reveals common features of snoRNA host genes. Mol Cell Biol 18: 6897-6909, 1998.

17. Mourtada-Maarabouni M, Hedge VL, Kirkham L, Farzaneh F and Williams GT: Growth arrest in human T-cells is controlled by the non-coding RNA growth-arrest-specific transcript 5 (GAS5). J Cell Sci 121: 939-946, 2008.

18. Mourtada-Maarabouni M, Pickard MR, Hedge VL, Farzaneh F and Williams GT: GAS5, a non-protein-coding RNA, controls apoptosis and is downregulated in breast cancer. Oncogene 28: 195-208, 2009 
19. Pickard MR and Williams GT: Molecular and cellular mechanisms of action of tumour suppressor GAS5 LncRNA. Genes (Basel) 6: 484-499, 2015.

20. Ehlicke F, Freimark D, Heil B, Dorresteijn A and Czermak P: Intervertebral disc regeneration: Influence of growth factors on differentiation of human mesenchymal stem cells (hMSC). Int J Artif Organs 33: 244-252, 2010.

21. Foss BL, Maxwell TW and Deng Y: Chondroprotective supplementation promotes the mechanical properties of injectable scaffold for human nucleus pulposus tissue engineering. J Mech Behav Biomed Mater 29: 56-67, 2014

22. Lewis BP, Burge CB and Bartel DP: Conserved seed pairing, often flanked by adenosines, indicates that thousands of human genes are microRNA targets. Cell 120: 15-20, 2005.

23. Friedman RC, Farh KK, Burge CB and Bartel DP: Most mammalian mRNAs are conserved targets of microRNAs. Genome Res 19: 92-105, 2009.

24. Livak KJ and Schmittgen TD: Analysis of relative gene expression data using real-time quantitative PCR and the 2(-Delta Delta $\mathrm{C}(\mathrm{T})$ ) method. Methods 25: 402-408, 2001.

25. Song J, Ahn C, Chun CH and Jin EJ: A long non-coding RNA, GAS5, plays a critical role in the regulation of miR-21 during osteoarthritis. J Orthop Res 32: 1628-1635, 2014.

26. Li F, Sun J, Huang S, Su G and Pi G: LncRNA GAS5 overexpression reverses LPS-induced inflammatory injury and apoptosis through up-regulating KLF2 expression in ATDC5 chondrocytes. Cell Physiol Biochem 45: 1241-1251, 2018.

27. Guo C, Song WQ, Sun P, Jin L and Dai HY: LncRNA-GAS5 induces PTEN expression through inhibiting miR-103 in endometrial cancer cells. J Biomed Sci 22: 100, 2015.

28. Zhao X, Wang P, Liu J, Zheng J, Liu Y, Chen J and Xue Y: Gas5 exerts tumor-suppressive functions in human glioma cells by targeting miR-222. Mol Ther 23: 1899-1911, 2015.

29. Wu Y, Lyu H, Liu H, Shi X, Song Y and Liu B: Downregulation of the long noncoding RNA GAS5-AS1 contributes to tumor metastasis in non-small cell lung cancer. Sci Rep 6: 31093, 2016.

30. Ma C, Shi X, Zhu Q, Li Q, Liu Y, Yao Y and Song Y: The growth arrest-specific transcript 5 (GAS5): A pivotal tumor suppressor long noncoding RNA in human cancers. Tumour Biol 37: 1437-1444, 2016.

31. Yu X and Li Z: Long non-coding RNA growth arrest-specific transcript 5 in tumor biology. Oncol Lett 10: 1953-1958, 2015.

32. Wang H, Liu H, Zheng ZM, Zhang KB, Wang TP, Sribastav SS Liu WS and Liu T: Role of death receptor, mitochondrial and endoplasmic reticulum pathways in different stages of degenerative human lumbar disc. Apoptosis 16: 990-1003, 2011.

33. Zhao CQ, Zhang YH, Jiang SD, Jiang LS and Dai LY: Both endoplasmic reticulum and mitochondria are involved in disc cell apoptosis and intervertebral disc degeneration in rats. Age (Dordr) 32: 161-177, 2010.

34. Gruber HE, Hoelscher GL, Bethea S and Hanley EN Jr: Mitochondrial membrane potential and nuclear and gene expression changes during human disc cell apoptosis: In vitro and in vivo annulus findings. Spine (Phila Pa 1976) 40: 876-882, 2015.
35. Ding F, Shao ZW, Yang SH, Wu Q, Gao F and Xiong LM: Role of mitochondrial pathway in compression-induced apoptosis of nucleus pulposus cells. Apoptosis 17: 579-590, 2012.

36. Yamada K, Sudo H, Iwasaki K, Sasaki N, Higashi H, Kameda Y, Ito M, Takahata M, Abumi K, Minami A and Iwasaki N: Caspase 3 silencing inhibits biomechanical overload-induced intervertebral disk degeneration. Am J Pathol 184: 753-764, 2014.

37. Gao J, Liu M, Zou Y, Mao M, Shen T, Zhang C, Song S, Sun M, Zhang S, Wang B, et al: Long non-coding RNA growth arrest-specific transcript 5 is involved in ovarian cancer cell apoptosis through the mitochondria-mediated apoptosis pathway. Oncol Rep 34: 3212-3221, 2015.

38. Liu JF, Chen CY, Chen HT, Chang CS and Tang CH: BL-038, a benzofuran derivative, induces cell apoptosis in human chondrosarcoma cells through reactive oxygen species/mitochondrial dysfunction and the caspases dependent pathway. Int J Mol Sci 17: pii: E1491, 2016.

39. Aziz MNM, Hussin Y, Che Rahim NF, Nordin N, Mohamad NE, Yeap SK, Yong CY, Masarudin MJ, Cheah YK, Abu N, et al: Curcumin analog DK1 induces apoptosis in human osteosarcoma cells in vitro through mitochondria-dependent signaling pathway. Molecules 23: pii: E75, 2018.

40. Chou WH, Liu KL, Shih YL, Chuang YY, Chou J,Lu HF, Jair HW, Lee MZ, Au MK and Chung JG: Ouabain induces apoptotic cell death through caspase- and mitochondria-dependent pathways in human osteosarcoma U-2 OS cells. Anticancer Res 38: 169-178, 2018.

41. Moriguchi Y, Alimi M, Khair T, Manolarakis G, Berlin C, Bonassar LJ and Härtl R: Biological treatment approaches for degenerative disk disease: A literature review of in vivo animal and clinical data. Global Spine J 6: 497-518, 2016.

42. Matta A, Karim MZ, Isenman DE and Erwin WM: Molecular therapy for degenerative disc disease: Clues from secretome analysis of the notochordal cell-rich nucleus pulposus. Sci Rep 7: 45623, 2017.

43. Wang Z, Perez-Terzic CM, Smith J, Mauck WD, Shelerud RA, Maus TP, Yang TH, Murad MH, Gou S, Terry MJ, et al: Efficacy of intervertebral disc regeneration with stem cells-a systematic review and meta-analysis of animal controlled trials. Gene 564: $1-8,2015$

44. Wang W, Deng G, Qiu Y, Huang X, Xi Y, Yu J, Yang X and Ye X: Transplantation of allogenic nucleus pulposus cells attenuates intervertebral disc degeneration by inhibiting apoptosis and increasing migration. Int J Mol Med 41: 2553-2564, 2018.

This work is licensed under a Creative Commons Attribution-NonCommercial-NoDerivatives 4.0 International (CC BY-NC-ND 4.0) License. 